\title{
High frequency Alfvén eigenmodes detected with ion-cyclotron-emission diagnostics during NBI and ICRF heated plasmas on the ASDEX Upgrade tokamak
}

R. Ochoukov ${ }^{1}$, R. Bilato ${ }^{1}$, V. Bobkov ${ }^{1}$, S. C. Chapman ${ }^{2}$, R. Dendy ${ }^{2,3}$, M. Dreval $^{4}$, H. Faugel ${ }^{1}$, A. Kappatou ${ }^{1}$, Ye. O. Kazakov ${ }^{5}$, M. Mantsinen ${ }^{6,7}$, K. G. McClements ${ }^{3}$, D. Moseev ${ }^{8}$, S. K. Nielsen $^{9}$, J.-M. Noterdaeme ${ }^{1,10}$, M. Salewski ${ }^{9}$, P. Schneider ${ }^{1}$, M. Weiland ${ }^{1}$, ASDEX Upgrade Team $^{\text {a) }}$, and MST1 Team ${ }^{\text {b) }}$

${ }^{1}$ Max Planck Institute for Plasma Physics, Boltzmannstr. 2, D-85748 Garching, Germany ${ }^{2}$ Centre for Fusion, Space and Astrophysics, University of Warwick, Coventry, CV4 7AL, United Kingdom

${ }^{3}$ Culham Centre for Fusion Energy, Culham Science Centre, Abingdon, Oxfordshire, OX14 3DB, UK

${ }^{4}$ Institute of Plasma Physics, National Science Center 'Kharkov Institute of Physics and Technology', Kharkov, Ukraine

${ }^{5}$ Laboratory for Plasma Physics, LPP-ERM/KMS, Brussels, Belgium

${ }^{6}$ Barcelona Supercomputing Center, Barcelona, Spain

${ }^{7}$ ICREA, Barcelona, Spain

${ }^{8}$ Max Planck Institute for Plasma Physics, Wendelsteinstr. 1, 17491 Greifswald, Germany

${ }^{9}$ Technical University of Denmark, Department of Physics, DK-2800 Kgs. Lyngby, Denmark

${ }^{10}$ Applied Physics Department, UGent, 9000 Gent, Belgium

a) see the author list in H. Meyer et al. 2019 Nucl. Fusion 59112014.

b) see the author list in B. Labit et al. 2019 Nucl. Fusion 59086020.

\begin{abstract}
The paper presents the first reported observation of high frequency Alfvén eigenmode excitation on the ASDEX Upgrade tokamak. The mode is driven in a novel way using radio frequency $(\mathrm{RF})$ wave acceleration of either beam-injected deuterium ions or thermal He-3 minority ions in a 3-ion heating scenario. In the case of beam ion acceleration, the instability only appears during deuteron acceleration at the $3^{\text {rd }}$ beam ion cyclotron harmonic (wave frequency $\omega=3 \Omega_{\mathrm{D}}$ where $\Omega_{\mathrm{D}}$ is the deuterium cyclotron frequency), as the mode is not detected during the more commonly used $2^{\text {nd }}$ harmonic / minority heating scenario or in the absence of beam-injected ions. The mode frequency is around $0.6-0.7 \Omega_{\mathrm{D}}$, where $\Omega_{\mathrm{D}}$ is evaluated in the low-field side plasma edge, and tracks the magnetic field $\mathrm{B}$ and the edge plasma electron density $\mathrm{n}_{\mathrm{e}}$ via the Alfvénic relation $\omega$ $\sim \mathrm{B} \mathrm{n}_{\mathrm{e}}^{-1 / 2}$. The mode does not appear as a single frequency wave but as a bundle of closely spaced (in frequency) sub-modes. When the parallel beam ion velocity component is increased, the sub-mode frequency spacing is observed to decrease, possibly due to a change in the eigenmode structure. Under certain conditions, typically in discharges with a relatively low plasma current, $\mathrm{I}_{\mathrm{P}}<0.7 \mathrm{MA}$, the mode appears to be driven directly by sub-Alfvénic deuterium beam ions. Absolute measurements of the mode amplitude show that at least $1 \%$ of the beam-injected power is transferred noncollisionally to the instability. While this is too low for practical alpha-channeling applications, discharges are planned with the aim of increasing the level of power transferred non-collisionally between fast ions and the instability.
\end{abstract}




\section{Introduction}

Stability and confinement of fusion born alpha particles is crucial for the overall performance of deuterium-tritium (DT) burning magnetized plasmas. As the alpha-particles resulting from thermonuclear DT fusion reactions are born with a narrow range of energies centred on about $3.5 \mathrm{MeV}$, they initially form an inverted population in velocity space, which tends to generate instabilities that may impact their confinement [1,2]. A particular class of instabilities occurs in the ion cyclotron frequency range, namely compressional Alfvén eigenmodes (CAEs) [3-5] and global Alfvén eigenmodes (GAEs) [5]. These high frequency instabilities are potentially important as they may play a role in channeling the energy from fusion born alpha-particles directly to bulk plasma fuel ions rather than heating the ions indirectly through Coulomb collisions with bulk electrons $[6,7]$. In plasmas with neutral beam injection (NBI), CAEs may also cause pitch angle scattering of the beam ions, thereby affecting the current driven by them [8].

Radio frequency (RF) emission with features that closely match those expected of CAEs and GAEs are commonly observed in NBI heated spherical tokamak plasmas [9-11, 5]. The low magnetic field strength ( $\sim 0.5 \mathrm{~T}$ or less) in spherical tokamaks implies that beam-injected ions (created with typical energies in the range of 50-100 keV) are super-Alfvénic. As a result, fast ion driven instabilities are excited much more readily. Examples of spherical tokamak plasmas where CAEs have been observed include START [9], NSTX [10] and MAST [11]. NSTX results include observations of mode mixing that is more consistent with GAEs than CAEs [5]. Under certain conditions CAEs can also be observed on DIII-D [12], which is a conventional tokamak. In this paper we present experimental results that extend the possible plasma conditions under which high frequency Alfvén eigenmodes can be excited in a conventional tokamak configuration. For the first time, we report high frequency instabilities driven by RFaccelerated ions. RF acceleration is achieved by externally launched fast ion cyclotron range of frequency (ICRF) waves. The particles undergoing acceleration were either beam-injected deuterium ions via their $3^{\text {rd }}$ cyclotron harmonic resonance $\left(3 \Omega_{\mathrm{D}}\right)$ in the plasma centre [13] or thermal He-3 minority ions via their fundamental resonance $\left(\Omega_{\mathrm{He} 3}\right)$ in a 3-ion plasma mixture [14]. In both cases the instabilities appear as periodic RF bursts, while the mode lasts as long as the power source is present, up to several seconds in duration. It suggests that anisotropy of the fast ion velocity distribution may be driving the mode, while the energy transfer is maintained non-collisionally via a predator-prey like cycle [15]: the instability (predator) periodically grows and removes the fast ion free energy (prey). Under certain conditions we also observe these modes driven directly by sub-Alfvénic beam-injected ions, as in the case of CAE observations on DIII-D [12]. Assuming that the mode in our case is a CAE makes it possible to calibrate its amplitude against a known source of coupled ICRF power from the antennas. The result shows that at least $1 \%$ of the NBI power is transferred non-collisionally to the mode. This amount is presently too low for practical alpha-channeling applications [6]. Future discharges are planned to increase the non-collisionally transferred power between beam-injected fast ions and the mode.

\section{Machine and Diagnostic description}

ASDEX Upgrade is a medium-sized tokamak (major radius $\mathrm{R}=1.67 \mathrm{~m}$, minor radius $\mathrm{a}=0.5$ $\mathrm{m})$ that nominally runs $\mathrm{H}$-mode discharges with the on-axis toroidal magnetic field $\mathrm{B}_{\mathrm{o}}$ ranging from $-1.8 \mathrm{~T}$ to $-2.5 \mathrm{~T}$ and the plasma current $\mathrm{I}_{\mathrm{P}}$ from 0.4 to $1.2 \mathrm{MA}$ [16]. The negative sign of the magnetic field indicates its toroidal direction in right-handed cylindrical $(R, \varphi, Z)$ 
coordinates; this is counter to the direction of the plasma current (Fig. 1). The machine is well equipped to study the phenomena of fast ion-driven instabilities: the auxiliary heating systems include ICRF heating (Fig. 1), NBI heating (Fig. 1), and electron cyclotron resonance heating (ECRH). The ICRF system can operate at frequencies of 30.0, 36.5, 41.8, and 55.1 MHz, while the NBI system can operate at $30-93 \mathrm{keV}$ beam injection energies. The beam injectors are oriented always in the co-current direction in the discharges used for this study (Fig. 1). The torus is extensively covered by high-frequency B-dot probes used to detect ICRF waves in the range of 1-100 MHz, either launched by the antennas or excited by the plasma itself [17]. For this study we use a low-field side probe in Sector 5 (ion cyclotron emission (ICE) Probe in Fig. 1), connected to a fast (up to $1.6 \mathrm{GHz}$ sampling rate) digitizer and an array of fast and slow wave probes in Sector 11 (Probe Array in Fig. 1). The signal from the ICE probe is used to measure frequency spectra of plasma modes. The signals from the probe array are acquired via a set of RF detectors, capable of measuring the RF wave amplitude and phase but not the frequency [18]. The ASDEX Upgrade tokamak is also equipped with a neutral particle analyzer (NPA) used to detect the presence of fast (50-200 keV) deuterons in the plasma [19].

\section{Experimental results and discussion}

CAEs and GAEs are generally (though not exclusively [12]) driven by super-Alfvénic fast ions. In the case of spherical tokamaks such ions are commonly generated via NBI [9-11, 5]. Since most of the existing NBI systems are currently used with a positive ion source, the energy of injected neutrals is limited to $\sim 100 \mathrm{keV}$ or less. As a result, the initial speed of beam-injected ions is usually sub-Alfvénic in a conventional tokamak plasma (where the magnetic field $\mathrm{B}$ > $1 \mathrm{~T}$ and plasma density $\mathrm{n}_{\mathrm{e}} \sim 2-6 \times 10^{19} \mathrm{~m}^{-3}$ ). Nevertheless, several methods exist that are capable of accelerating plasma ions to high energies ( $1 \mathrm{MeV}$ or higher), driving them well above the Alfvén speed. Such mechanisms are the subject of the following section.

\subsection{High frequency Alfvén eigenmode observations in discharges with RF-accelerated ions}

One such method utilizes externally-launched ICRF waves to accelerate beam-injected ions perpendicular to the magnetic field via the $3^{\text {rd }}$ beam ion cyclotron harmonic $\left(\omega=3 \Omega_{D}\right)$ [13]. Fig. 2 shows an example of such an acceleration mechanism for the case of ASDEX Upgrade, where the acceleration process is accompanied by emission the frequency of which closely follows the Alfvénic dependence $\sim \mathrm{B} \mathrm{n}_{\mathrm{e}}{ }^{-1 / 2}$. Possible candidates for the observed instability are the CAE and the GAE. In the discharge shown in Fig. 2, the ICRF power is launched in 6 pulses, each $0.5 \mathrm{~s}$ long and spaced apart by $0.5 \mathrm{~s}$ (Fig. 2 (d) and (e)). The deuterium discharge is an $\mathrm{H}-$ mode, maintained by $2.5 \mathrm{MW}$ of deuterium NBI power (full energy $\mathrm{E}_{\mathrm{NBI}}=59 \mathrm{keV}$, Fig. 2 (f)) and 1.7 MW of ECRH power. The on-axis magnetic field $\mathrm{B}_{\mathrm{o}}$ is $-2.5 \mathrm{~T}$ and the plasma current $\mathrm{I}_{\mathrm{P}}$ is 0.6 MA. The sequence of the six ICRF pulses is as follows: \#1) the power is launched at $36.5 \mathrm{MHz}$, which corresponds to the second cyclotron harmonic of the deuterium beam ions ( $\omega$ $=2 \Omega_{\mathrm{D}}$ ) in the plasma center; \#2) the same power is launched at $55.1 \mathrm{MHz}$, which corresponds to the third cyclotron harmonic of the deuterium beam ions $\left(3 \Omega_{D}\right)$ in the plasma center; \#3) and $\# 4$ ) both the $2^{\text {nd }}$ and the $3^{\text {rd }}$ harmonics are applied simultaneously at two different power levels; and 5\#) and 6\#) only the $3^{\text {rd }}$ harmonic is launched at constant power and the NBI power is varied. The effect of applying the $2^{\text {nd }}$ and $3^{\text {rd }}$ cyclotron harmonics on the fast deuterium ions is visible on both the neutron rate N (Fig. 2 (c)) and the NPA count rate (Fig. 2 (g)). The change in the neutron rate during ICRF pulse \#2 (when $3 \Omega_{\mathrm{D}}$ is applied) is more than double that 
observed during ICRF pulse \# 1 (when $2 \Omega_{\mathrm{D}}$ is applied), even though the applied ICRF power in \#2 (1.7 MW) is only $31 \%$ larger than in \#1 (1.3 MW). The NPA spectra view pitch values of 0.5-0.7 and reveal that the application of $2 \Omega_{\mathrm{D}}(\mathrm{t}=3.2-3.5 \mathrm{~s})$ has a strong effect at the lower energies, $<100 \mathrm{keV}$, while the application of $3 \Omega_{\mathrm{D}}(\mathrm{t}=4.2-4.5 \mathrm{~s})$ has a stronger effect for energies $>100 \mathrm{keV}$ (see the highlighted regions in Fig. $2(\mathrm{~g})$ ). Note that the value of the Alfvén speed in the low-field side plasma edge region (at the normalized poloidal flux coordinate $\rho_{p} \sim 0.7-0.9$, where modes such as the CAEs are expected to exist [4]) is $\sim 6.9 \times 10^{6} \mathrm{~m} \mathrm{~s}^{-1}$, which is the speed of a deuteron with energy $\sim 500 \mathrm{keV}$, outside the NPA detection range [19].

High frequency spectra obtained with the ICE diagnostic clearly show the presence of the applied ICRF waves at $36.5 \mathrm{MHz}$ and 55.1 MHz (Fig. 3 (a)). However, under certain conditions, we also detect a band (or group of modes) of closely spaced peaks in the 9-10 $\mathrm{MHz}$ frequency range (Fig. 3 (b)). This frequency value corresponds to $\sim 0.6-0.7 \Omega_{\mathrm{D}}$ in the low-field side edge plasma region, where we also detect beam-driven ion cyclotron emission at $\sim 14 \mathrm{MHz}$ (NBI ICE in Fig. 3 (a)). The NBI-driven ICE is identified via frequency matching [20]. The lower frequency modes only appear during the application of $55.1 \mathrm{MHz}\left(3 \Omega_{\mathrm{D}}\right)$, strictly in the presence of beam-injected ions: no signal above the noise level is observed during ICRF pulse \#1 $\left(2 \Omega_{D}\right.$ only), or in the absence of beam-injected ions. However, the presence of the $2 \Omega_{\mathrm{D}}$ harmonic does not appear to impede the 9-10 MHz modes when they are excited by the application of $3 \Omega_{\mathrm{D}}$ harmonic in ICRF pulses \#3 and \#4 (Fig. 3 (b)). When the applied ICRF power at $3 \Omega_{\mathrm{D}}$ is kept constant and the NBI power is increased, the overall mode intensity increases, while the frequency spacing between adjacent peaks decreases from $\sim 120 \mathrm{kHz}$ to $\sim 100 \mathrm{kHz}$ (ICRF pulses \#5 and \#6 in Fig. 3 (c)). Note that the last NBI power step (7.5-8.7 s) applies the beam with the same pitch but with the full energy $E_{\mathrm{NBI}}=92 \mathrm{keV}$. The observed frequency response may be due to the change in the eigenmode structure, as the edge plasma density rises slightly between steps \#5 and \#6 (Fig. 2 (b)). An additional minor effect on the mode frequency spacing is expected from the co-current toroidal plasma rotation frequency increase from $4.8 \mathrm{kHz}$ to 5.6 $\mathrm{kHz}$.

The mode is also observed when the same plasma scenario is repeated but at reduced $\mathrm{E}_{\mathrm{NBI}}=45$ $\mathrm{keV}$ (Fig. 4), although the mode intensity is reduced (ICRF pulses \#3 and \#5) and, in some cases, (ICRF pulse \#6) is too weak to be visible above the noise level (Fig. 4 (g)). A slight rise in the mode frequency is observed, compared to Fig. 2. Our observations are in contrast to those first reported on NSTX [10], where the parallel beam ion velocity component is varied via a pitch change but no change in the CAE mode frequency is observed. The NSTX results [10] suggest that the intrinsic mode frequency is a global parameter set by the background plasma. On the other hand, comparison with the CAE frequency values observed on DIII-D (see Fig. 3, bottom plot in [12]) produces a good match with our results, when we linearly rescale the values with the magnetic field strength. Following the theoretical estimates by Gorelenkov et al. [21] toroidal mode numbers are expected to produce the smallest frequency splitting values (see Eq. (5) in [21]). Therefore, the DIII-D values for the CAE frequency $(\sim 2.5 \mathrm{MHz})$ and frequency splitting $(\sim 20-30 \mathrm{kHz})$ at $0.6 \mathrm{~T}$ rescale to $\sim 10 \mathrm{MHz}$ and $\sim 80-120 \mathrm{kHz}$ at $2.5 \mathrm{~T}$ for our case. These values are a close match to the values we observe in Figs. 3 (b) and 4 (g). Unlike the DIII-D results, where three different frequency splitting dependencies (radial, poloidal, and toroidal) are clearly observed, we only detect a single band of modes. This makes the identification of our mode as a CAE less definitive. 
The ICRF system on ASDEX Upgrade is also capable of operating at $41.8 \mathrm{MHz}$, which makes it possible to reproduce the mode appearance via $3 \Omega_{\mathrm{D}}$ beam ion acceleration in discharges at lower magnetic field $\mathrm{B}_{\mathrm{o}}=-2.0 \mathrm{~T}$. In this case, the mode peaks appear centered at $\sim 7.5 \mathrm{MHz}$ (Fig. 5): the large scale mode frequency drop from $\sim 9.5 \mathrm{MHz}$ at $\mathrm{B}_{\mathrm{o}}=-2.5 \mathrm{~T}$ to $\sim 7.5 \mathrm{MHz}$ at $\mathrm{B}_{\mathrm{o}}$ $=-2.0 \mathrm{~T}$ is well described by the Alfvénic dependence $\omega \sim \mathrm{B} \mathrm{n}_{\mathrm{e}}^{-1 / 2}$ and almost entirely accounted for by the magnetic field change. Small scale mode frequency changes observed during a single discharge (when the magnetic field is kept constant) are also well described by the Alfvénic dependence (Fig. 5 (b)): in this case the mode frequency varies due to the changes in the edge plasma density rather than the magnetic field.

In all cases, the mode appearance is not prompt, but typically takes $\sim 0.05-0.15 \mathrm{~s}$ to develop after the ICRF power is switched on (Figs. 5 (b) and 6 (b)): the time is comparable to how long it takes for the neutron rate to reach its steady-state value (Fig. 2 (c)), which points at a particle origin of the mode driver, rather than being driven directly by the launched ICRF wave. As mentioned previously, the mode appears not as a single peak but as a collection of closely spaced (in frequency) peaks. The frequency response of the peaks to changes of the parallel beam ion velocity component (Fig. 3 (c)) suggests that frequency splitting is due to the toroidal mode number $\mathrm{n}$, with the modes travelling in the counter-current direction. Interestingly, as the ICRF power (operated at $3 \Omega_{\mathrm{D}}$ ) is increased (while keeping the beam-injected power constant), the total instability intensity increases via the excitation of additional modes propagating in both the co- and, to a greater extent, the counter-current directions (Figs. 6 (b) and (c), where the toroidal mode number $\mathrm{n}$ is relative to the dominant mode). The modes propagating in the co-current direction have the shortest growth time and the maximal number of these modes appears limited (Fig. 6 (b)). The mode frequency dependence on $\mathrm{n}$ is weaker than linear, even after the measured frequency is corrected for the Doppler shift due to the toroidal plasma rotation $\left(\mathrm{nf}_{\phi}\right)$ (Fig. $6(\mathrm{~d})$ ), and is well described by a decaying exponential: $\mathrm{f}+\mathrm{nf}_{\phi}=11.8-$ $2.1 \mathrm{e}^{-0.05 \mathrm{n}}[\mathrm{MHz}]$. The decay time of the mode intensity after the ICRF power is switched off follows the growth rate trend: the peaks with the highest observed frequencies are the first to stabilize, in less than $1 \mathrm{~ms}$, while the lowest frequency modes take much longer to decay away (Fig. 7).

It is also possible to RF-accelerate thermal, rather than beam-injected, ions to super-Alfvénic energies. One such mechanism currently studied on ASDEX Upgrade and elsewhere is the three-ion heating scheme [14]. The plasma scenario on ASDEX Upgrade uses a trace $(\leq 1 \%)$ amount of $\mathrm{He}-3$, in combination with $80 \% \mathrm{H}$ and $20 \% \mathrm{D}$ plasma mix, which allows for an efficient power transfer from the launched fast wave to the He-3 ions. In this case, ICRF heating is also accompanied by a mode at $\sim 10 \mathrm{MHz}$ (Fig. 8). A close examination of the mode dynamics shows that the emission is bursty in nature (Fig. 8 (d)): the cyclical nature of the instability is discussed in more detail next in the context of beam-driven emission.

\subsection{Observation of high frequency Alfvén eigenmodes driven by sub-Alfvénic beam ions}

Although high frequency Alfvén eigenmodes, such as the CAEs and GAEs are much more readily excited by super-Alfvénic ions, excitation via sub-Alfvénic beam ions has been previously reported on DIII-D [12]. This is also observed in some low plasma current discharges (<0.7 MA) on ASDEX Upgrade (Fig. 9). The mode frequency (7.5-8.0 MHz, Fig. 9 (a)) nearly matches the results shown in Fig. 5, as both plasmas have $\mathrm{B}_{\mathrm{o}} \sim-2.0 \mathrm{~T}$ and similar edge plasma density $\sim 2.8 \times 10^{19} \mathrm{~m}^{-3}$. The mode frequency changes observed during the 
discharge are due to the edge plasma density variation, as expected from the Alfvénic nature of the mode: $\omega_{\mathrm{AE}} \sim \mathrm{B} \mathrm{n}_{\mathrm{e}}^{-1 / 2}$. Since no ICRF power is applied in the case of Fig. 9, the probe signal is dominated by the mode emission. As a result, it is now possible to deduce more information on the mode properties via the probe array in Sector 11 (Fig. 1). We choose the time period where frequency splitting is minimal (Fig. 9 (b)) to ensure that beat waves have no impact on the phase (and, hence, $k_{/ /}$) measurement of the mode. As previously noted, the mode emission is not uninterrupted but appears in bursts (Fig. 9 (c)): the mode itself typically lasts for $\sim 0.1 \mathrm{~ms}$ and the duration between consecutive bursts is generally $\sim 1 \mathrm{~ms}$. Such bursty dynamics has been observed previously on DIII-D for the case of CAEs [15] and is attributed to a predator-preytype behavior of the instability, whereby the mode (predator) periodically removes free energy from the fast ion population (prey). The signal strength detected by the probes oriented to measure the fast wave (FW) remains consistently above the slow wave (SW) signal strength (Fig. 9 (d)), strongly suggesting the compressional nature of the mode (or the CAE). However, the possibility of the shear (slow) wave mode origin (or the GAE) cannot be definitively excluded due to the edge probe measurements being susceptible to shear-compressional polarization mixing $[22,23]$. The phase measurement between neighboring $\mathrm{FW}$ probes also remains robust (Fig. 9 (e)), which allows us to reconstruct the magnitude of the mode's parallel wavenumber $\left|k_{/}\right|=21.5 \mathrm{~m}^{-1}$ (Fig. 10). For comparison, the ASDEX Upgrade ICRF antennas launch fast waves with $\left|k_{/ /}\right|=8-10 \mathrm{~m}^{-1}$. The large magnitude of the measured $k_{/ /}$value makes it difficult to identify the mode as either a CAE or a GAE.

\subsection{Applicability to non-collisional particle-wave power transfer (alpha channeling)}

One notable feature of the mode is its duration: it generally lasts for as long as the power source, such as ICRF and NBI, is present in the plasma. This feature makes it potentially relevant to future fusion reactors that will operate in steady-state. A record long NBI-driven mode (over 8 $\mathrm{s}$ in duration) is shown in Fig. 11. The mode lasts far longer than the beam ion slowing down time, strongly suggesting that it is the anisotropy of the fast ion velocity space that continuously drives the mode [12]. A TRANSP-reconstructed [24] beam ion velocity distribution function $\mathrm{f}\left(\mathrm{E}\right.$, Pitch), at $\rho_{\text {tor }}=0.7-0.8$ along the midplane on the low-field side, reveals that strong pitch angle gradients are maintained throughout the discharge (Fig. 12). We can also see that the local Doppler-shifted cyclotron resonance condition (Equation (8) in [25]) marginally intercepts the dominant beam ion distribution function (Fig. 12) for our particular mode frequency (10.8 $\mathrm{MHz}$ ) and parallel wavenumber $\left(21.5 \mathrm{~m}^{-1}\right)$, providing further support to anisotropy as the mode driver. As we noted earlier (Fig. 9), the energy exchange between the fast ions and the mode is not constant but follows a collisionless predator-prey-type cycle, as described in detail for the case of CAEs on DIII-D [15]. Assuming that our mode is also a CAE, we can deduce how much energy is transferred non-collisionally from beam-injected ions to the mode by launching a known amount of ICRF power from the antennas. A pair of $1 \mathrm{~s}$ long, $3.2 \mathrm{MW} \mathrm{ICRF}$ power pulses (operated at $36.5 \mathrm{MHz}$ or $2 \Omega_{\mathrm{D}}$ beam ion harmonic) was applied as a calibrated power source (Fig. 11 (a)): the ICRF pulses do not interrupt the mode (Fig. 11 (b)). Integrating the probe signal over the time interval when ICRF was on (2-3 s) and comparing it with the signal integral when only the mode was present (3-4 s) reveals a ratio of $\sim 100$. Since the ICRF power pulse operated at 3.2 MW of power and delivered 3.2 MJ of energy, there were $32 \mathrm{~kJ}$ of energy contained in the mode during a 1 second interval. Since the mode is driven by $2.5 \mathrm{MW}$ on NBI power, at least $1 \%$ of the NBI power was transferred to the mode non-collisionally. The calculated mode power is consistent across all the fast wave probes inside the ASDEX Upgrade torus. We acknowledge that our method does not account for the direction of the RF power flow 
from the mode to the probe being the opposite of that from the active antennas. Nor do we account for the different RF power damping mechanisms. However, these shortcomings would increase our estimated NBI-mode power transfer meaning that our original $1 \%$ estimate is a reasonable lower bound. Although this amount is currently too small for practical alphachanneling applications [6], discharges are planned with the aim of increasing the amount of power transferred non-collisionally between beam-injected fast ions and the mode. The beamdriven mode appearance for low IP values suggests a further mode amplitude optimization via the edge safety factor increase.

\section{Summary}

The paper presents a first extensive set of observations of high frequency Alfvén eigenmode emission on ASDEX Upgrade, a conventional tokamak device. The mode is readily excited in discharges where beam-injected ions are RF-accelerated to high energies at the third $\left(3 \Omega_{\mathrm{D}}\right)$ beam ion cyclotron harmonic (Figs. 3-6). The mode is not observed during RF acceleration of the beam ions at $2 \Omega_{D}$, nor does the presence of ICRF power at $2 \Omega_{D}$ in addition to $3 \Omega_{D}$ impede/enhance the mode (Figs. 3 and 4). The mode is also not excited by ICRF power at $3 \Omega_{D}$ in the absence of beam-injected ions. The mode frequency is $\sim 0.6-0.7 \Omega_{\mathrm{D}}$ where $\Omega_{\mathrm{D}}$ is evaluated in the low-field side plasma edge and follows the Alfvénic dependence $\omega_{\mathrm{A}} \sim \mathrm{B} \mathrm{n}^{-1 / 2}$ when either the magnetic field or the edge plasma density is varied (Figs. 5 and 6). When excited, the mode does not appear at a single frequency value but exists as a bundle of closely spaced peaks. The mode frequency spacing is observed to drop, when the parallel beam ion velocity component is increased, which may be due to a change in the eigenmode structure. The mode is also observed when thermal, rather than beam-injected, ions are efficiently accelerated to high energies, for example, in discharges heated via the 3-ion scheme (Fig. 8). Interestingly, we also observe the mode excited by the (sub-Alfvénic) beam ions directly, typically in discharges with a low plasma current, $\mathrm{I}_{\mathrm{P}}<0.7 \mathrm{MA}$ (Figs. 9 and 11). In all cases, the mode persists for as long as the relevant heating power remains active, either the NBI or the ICRF or both, generally longer than the fast ion slowing down time via Coulomb collisions (Figs. 5, 6, 8, 9, and 11). This points at the fast ion velocity distribution anisotropy as the mode driver, with the local Doppler-shifted resonance condition marginally intercepting the beam ion velocity distribution (Fig. 12). The energy exchange between the mode and the fast ion is not continuous but follows a predator-prey like cycle. Such temporal behavior is observed in discharges with RFaccelerated ions (Fig. 8) and sub-Alfvénic beam-injected ions (Figs. 9 and 11). The duration of each emission burst is typically $\sim 0.1 \mathrm{~ms}$ while the cycle period is $\sim 1 \mathrm{~ms}$ long (Fig. 9 (c)). Measurements from the field-aligned array of fast and slow wave probes indicates that the fast wave polarization dominates (Fig. 9 (d)), which would suggest that the mode is compressional. The magnitude of the parallel mode wavenumber is $|k /|=21.5 \mathrm{~m}^{-1}$, about double the size of a typical fast wave launched by the ASDEX Upgrade ICRF antennas (Fig. 10). Assuming that the mode is compressional and calibrating its intensity against a known amount of ICRF power coupled by the antennas shows that at least $1 \%$ of NBI power is transferred to the instability non-collisionally (Fig. 11 (d)). Although the amount is currently too small for practical alphachanneling applications, future discharges are planned with the aim of increasing the power fraction transferred non-collisionally between fast ions and the mode.

\section{Acknowledgements}

This work has been carried out within the framework of the EUROfusion Consortium and has received funding from the Euratom research and training programme 2014-2018 and 2019-2020 
under grant agreement No 633053. The views and opinions expressed herein do not necessarily reflect those of the European Commission. Wei Zhang's contribution to the development of NBI heated discharges presented in this manuscript is acknowledged.

\section{References}

[1] Kolesnichenko, Ya. I., Sov. J. Plasma Phys. 6 (5), Sept.-Oct. 1980, p. 531-532.

[2] J.G. Cordey, R.J. Goldston, D.R. Mikkelsen, Nucl. Fusion, Vol. 21, No. 5 (1981).

[3] Mahajan S.M. and Ross D.W. 1983 Phys. Fluids 262561.

[4] B. Coppi et al. Physics of Fluids 29, 4060 (1986).

[5] N. N. Gorelenkov, et al., Nucl. Fusion 43, 228-233 (2003).

[6] N. J. Fisch, Phys. Plasmas 2, 6 (1995).

[7] E. V. Belova, N. N. Gorelenkov, E. D. Fredrickson, and K. Tritz, Phys. Rev. Lett. 115, 015011 (2015).

[8] H. J. C. Oliver et al 2014 Plasma Phys. Control. Fusion 56125017.

[9] K. G. McClements et al. Plasma Phys. Control. Fusion 41, 661-678 (1999).

[10] E. D. Fredrickson et al. Phys. Rev. Lett. 87, 14 (2001).

[11] L. C. Appel et al. Plasma Physics and Controlled Fusion 50, 11 (2008).

[12] W. W. Heidbrink et al. Nuclear Fusion 46, 324 (2004).

[13] M. J. Mantsinen et al. Phys Rev. Lett., 88, 10 (2002).

[14] Ye. O. Kazakov et al., Nature Physics 13, 973-978 (2017).

[15] W. W. Heidbrink and J. R. Danielson, Physics of Plasmas 1, 4120 (1994).

[16] H. Meyer et al. 2019 Nucl. Fusion 59112014.

[17] R. Ochoukov et al. EPJ Web of Conferences 157, 03038 (2017).

[18] R. Ochoukov et al. Review of Scientific Instruments 86, 115112 (2015).

[19] P. A. Schneider et al. Review of Scientific Instruments 86, 073508 (2015).

[20] R. Ochoukov et al. Review of Scientific Instruments 89, 10J101 (2018).

[21] N. N. Gorelenkov et al. Nuclear Fusion 42, 977 (2002).

[22] E. V. Belova et al., Physics of Plasmas 24, 042505 (2017).

[23] E. D. Fredrickson et al. Nucl. Fusion 52, 043001 (2012).

[24] https://transp.pppl.gov (Version 18.2) DOI: 10.11578/dc.20180627.4.

[25] S. E. Sharapov, M. K. Lilley, R. Akers, N. Ben Ayed, M. Cecconello, J. W. S. Cook, G. Cunningham, E. Verwitchte, Phys. Plasmas 21, 082501 (2014). 


\section{Figures}

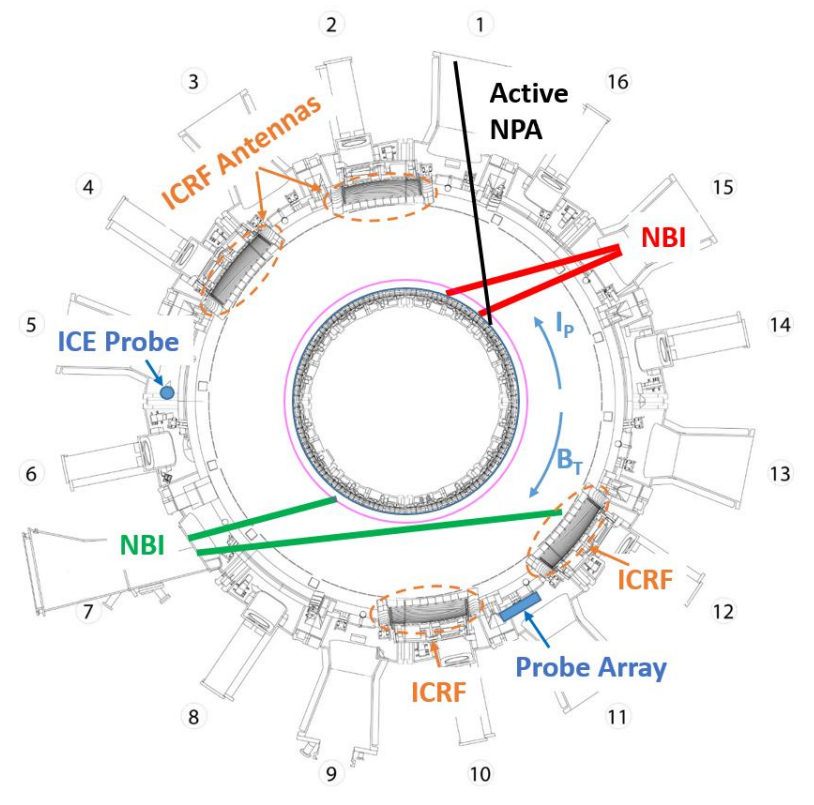

Fig. 1: Top cross-sectional view of the ASDEX Upgrade torus. The structures shown are ICRF antennas in Sectors 2, 4, 10, and 12; NBI boxes in sectors 7 and 15; ICE probe in Sector 5; active NPA line of sight; and probe array in Sector 11. 

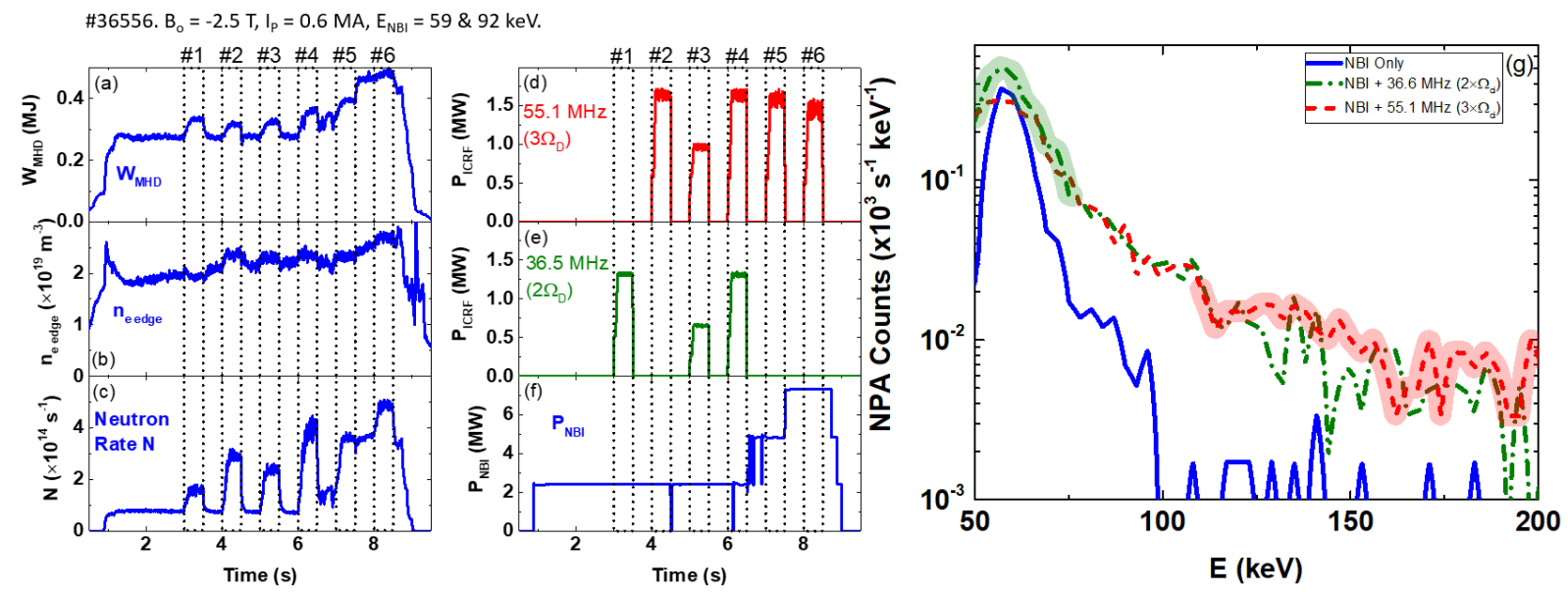

Fig. 2: Key plasma parameters in an ASDEX Upgrade discharge (\#36556) demonstrating high frequency Alfvén eigenmode excitation in the presence of RF-accelerated fast ions via the $3^{\text {rd }}$ beam ion cyclotron harmonic resonance $\left(3 \Omega_{D}\right)$. (a) the plasma stored energy $\mathrm{W}_{\mathrm{MHD}}$; (b) the plasma edge density $\mathrm{n}_{\mathrm{e}}$ edge; (c) the neutron rate $\mathrm{N}$; (d) the ICRF power $\mathrm{P}_{\text {ICRF }}$ launched at 55.1 $\mathrm{MHz}$ (corresponding to $3 \Omega_{\mathrm{D}}$ in the plasma centre); (e) the ICRF power launched at $36.5 \mathrm{MHz}$ (corresponding to $2 \Omega_{\mathrm{D}}$ in the plasma centre); (f) the NBI power $\mathrm{P}_{\mathrm{NBI}}$ launched at primary beam energy of $59 \mathrm{keV}$, with the exception of the last NBI power step (7.5-8.7 s) which was at 92 $\mathrm{keV}$; and (g) the NPA spectral counts for deuterons, $\mathrm{t}=3.7-4.0 \mathrm{~s}$ (NBI only), $\mathrm{t}=3.2-3.5 \mathrm{~s}$ (NBI $+36.5 \mathrm{MHz})$, and $\mathrm{t}=4.2-4.5 \mathrm{~s}(\mathrm{NBI}+55.1 \mathrm{MHz})$; the slowing down times for $200 \mathrm{keV}$ deuterons range from $100 \mathrm{~ms}$ in the edge to $250 \mathrm{~ms}$ in the core plasma. The on-axis magnetic field $B_{0}$ is $-2.5 \mathrm{~T}$ and the plasma current $\mathrm{I}_{\mathrm{P}}$ is $0.6 \mathrm{MA}$. 

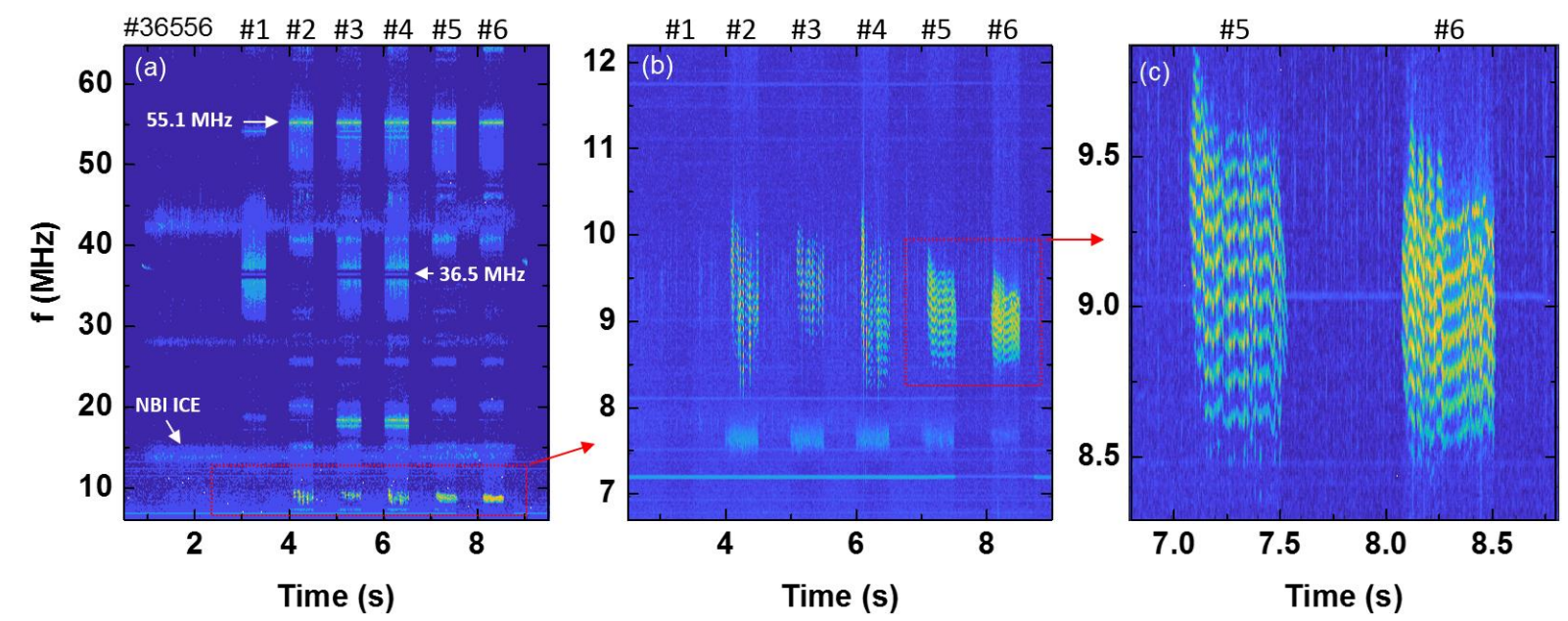

Fig. 3: (a) High frequency spectra obtained with the ICE diagnostic for the discharge shown in Fig. 2: NBI ICE refers to beam-driven ICE in the low-field side plasma edge; (b) a close-up view of the high frequency Alfvén eigenmode emission spectra; and (c) a close-up view of the mode spectra during an increase in $\mathrm{P}_{\mathrm{NBI}}$. 

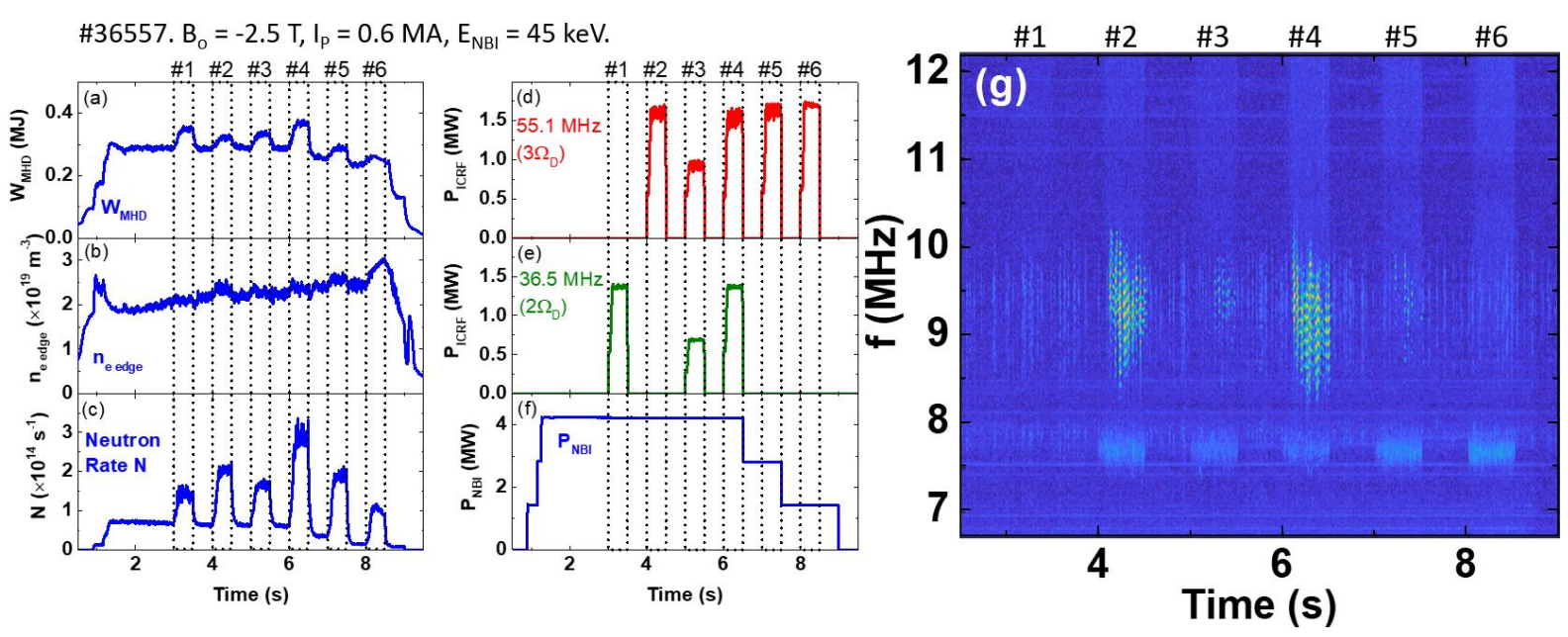

Fig. 4: List of key plasma parameters in an example discharge (\#36557) used to demonstrate high frequency Alfvén eigenmodes in the presence of RF-accelerated fast ions via $3^{\text {rd }}$ beam ion cyclotron harmonic $\left(3 \Omega_{D}\right)$ with a reduced NBI energy. (a) the plasma stored energy $\mathrm{W}_{\mathrm{MHD}}$; (b) the plasma edge density $\mathrm{n}_{\mathrm{e}}$ edge; (c) the neutron rate $\mathrm{N}$; (d) the ICRF power PICRF launched at $55.1 \mathrm{MHz}$ (corresponding to $3 \Omega_{\mathrm{D}}$ in the plasma centre); (e) the ICRF power launched at 36.5 $\mathrm{MHz}$ (corresponding to $2 \Omega_{\mathrm{D}}$ in the plasma centre); (f) the NBI power $\mathrm{P}_{\mathrm{NBI}}$ launched at primary beam energy of $45 \mathrm{keV}$ for all power steps; and (g) a close-up view of the mode spectra during the discharge. The on-axis magnetic field $\mathrm{B}_{\mathrm{o}}$ is $-2.5 \mathrm{~T}$ and the plasma current $\mathrm{I}_{\mathrm{P}}$ is $0.6 \mathrm{MA}$. 

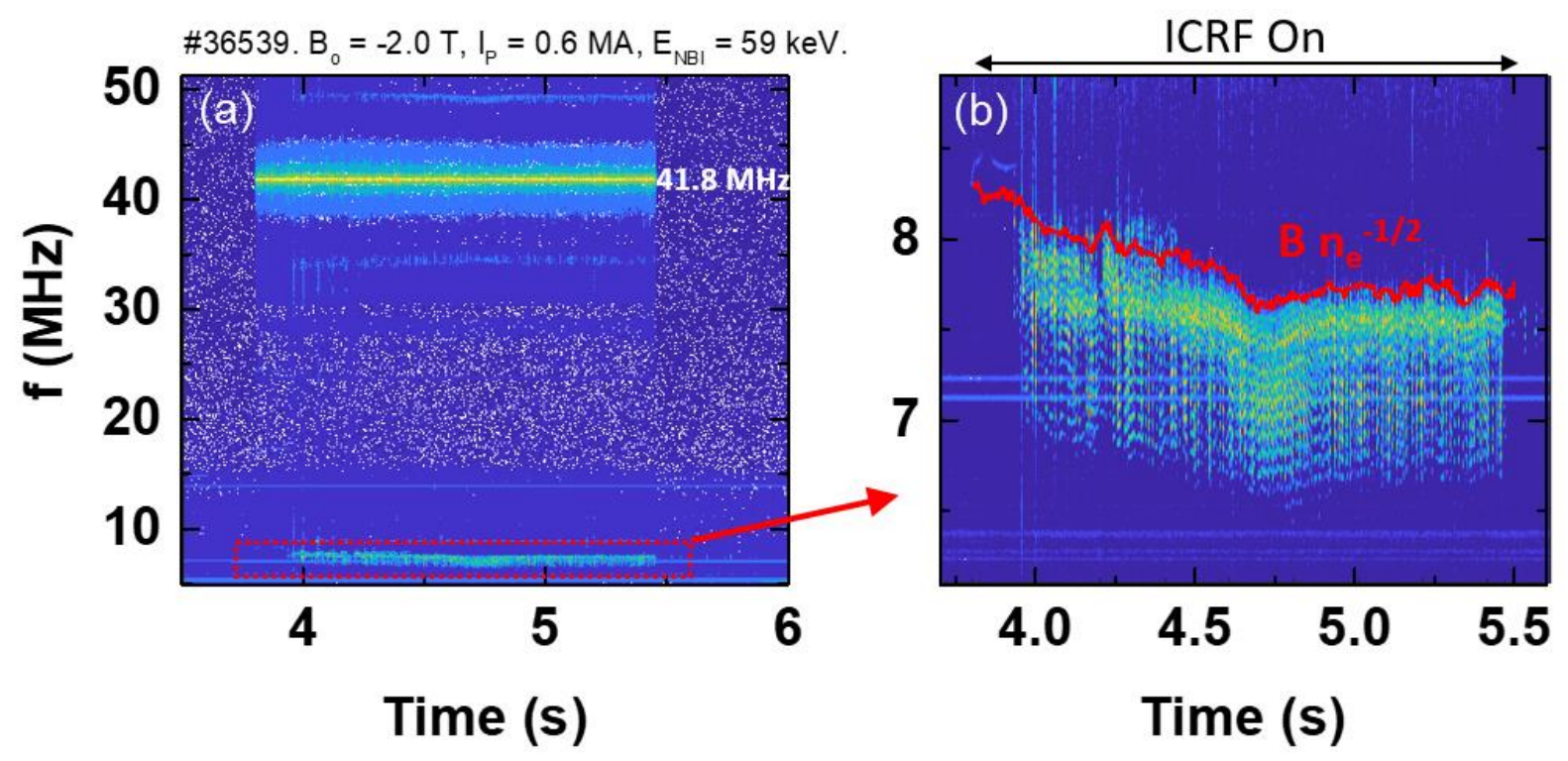

Fig. 5: Demonstration of high frequency Alfvén eigenmodes in the presence of RF-accelerated fast ions via the $3^{\text {rd }}$ beam ion cyclotron harmonic resonance $\left(3 \Omega_{D}\right)$ in a discharge with a reduced (and constant) magnetic field $\mathrm{B}_{\mathrm{o}}=-2.0 \mathrm{~T}$. The ICRF power is launched at $41.8 \mathrm{MHz}$. (a) High frequency spectra obtained with the ICE diagnostic; and (b) a close-up view of the mode spectra during the discharge. The red line in (b) indicates the Alfvénic frequency trend $\omega \sim B \mathrm{n}^{-1 / 2}$, rescaled to match the maximum mode frequency. 

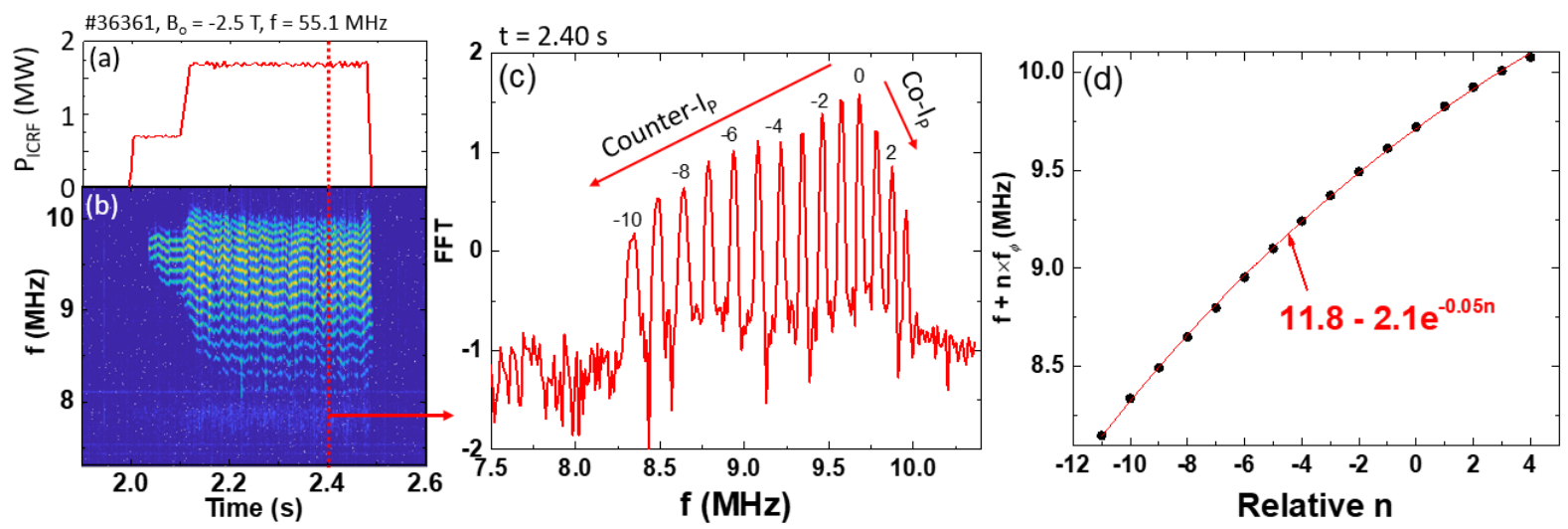

Fig. 6: A detailed spectral view of the mode and its response to ICRF power change. (a) The ICRF power launched at $55.1 \mathrm{MHz}$ (corresponding to $3 \Omega_{\mathrm{D}}$ in the plasma center). (b) A closeup view of the mode frequency spectra. (c) A fast Fourier Transform (FFT) of the mode under steady-state conditions; the integers labelling the peaks indicate the relative toroidal mode number $n$. Decreasing (increasing) mode frequencies are labeled as counter-IP (co-IP), with respect to the dominant mode. (d) The mode frequency spectra in the plasma frame, accounting for the Doppler shift due to the co-current toroidal plasma rotation $\left(n \times f_{\phi}\right)$. The mode frequency dependence on the mode number is weaker than linear. The NBI power is constant at $5 \mathrm{MW}$. 


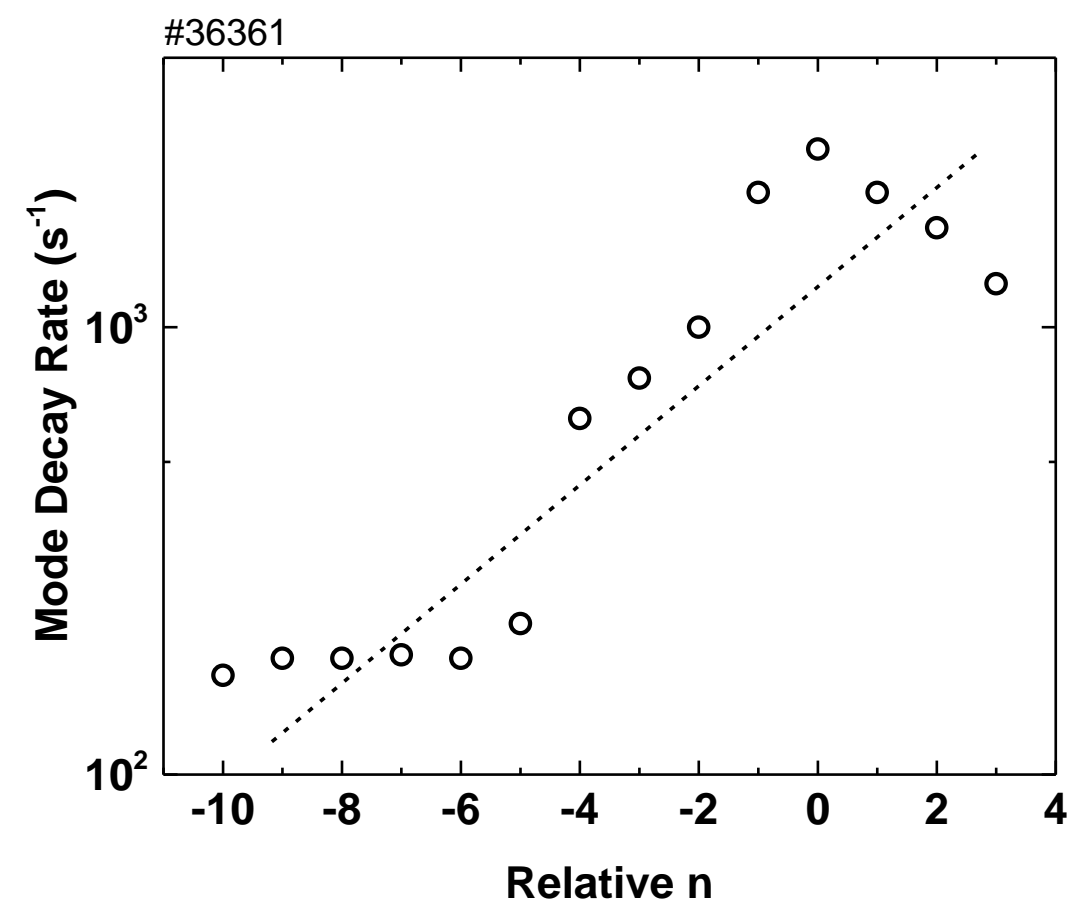

Fig. 7: The decay rate of various mode peaks after the ICRF power is switched off. The straight dashed line is to guide the eye only. 


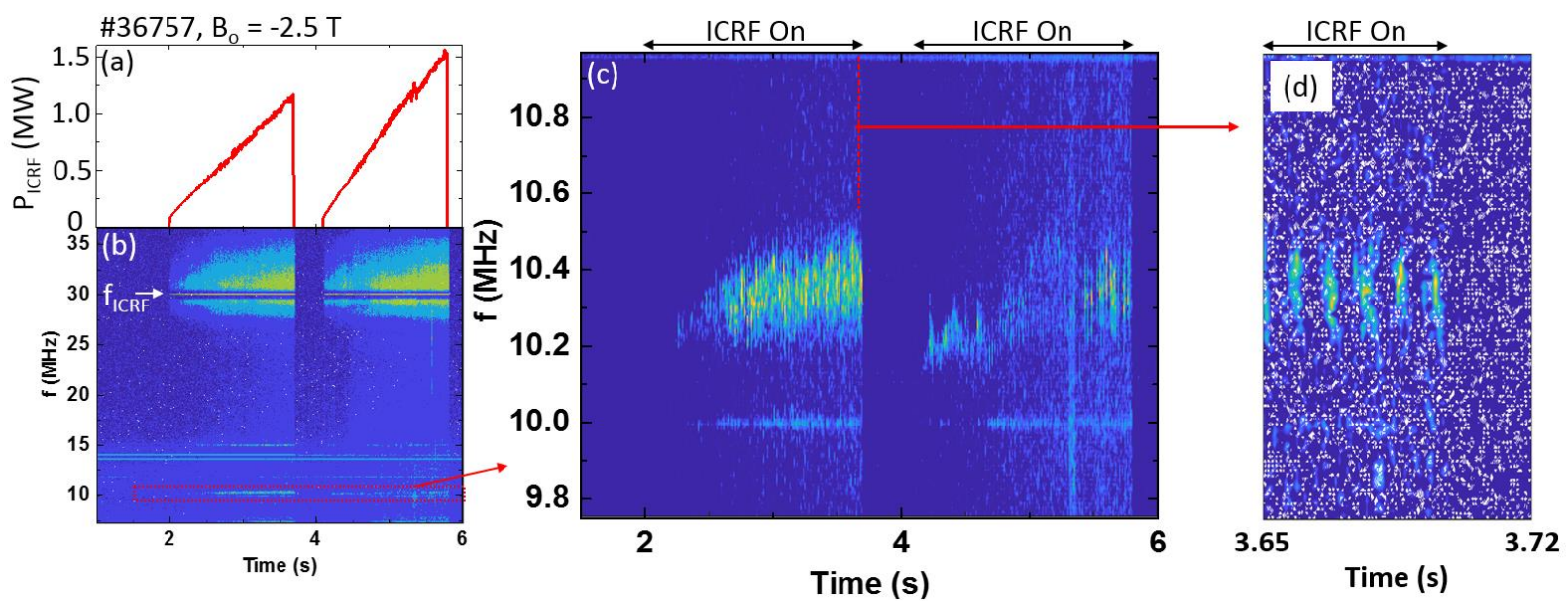

Fig. 8: An example discharge (\#36757) that utilizes the 3-ion heating scheme to generate energetic He-3 ions. (a) The coupled ICRF power PICRF; (b) frequency spectra measured with the ICE diagnostic; (c) a close-up view of the mode that appears in the presence of accelerated He-3 ions; and (d) a detail of the time evolution that shows the bursty nature of the mode. 

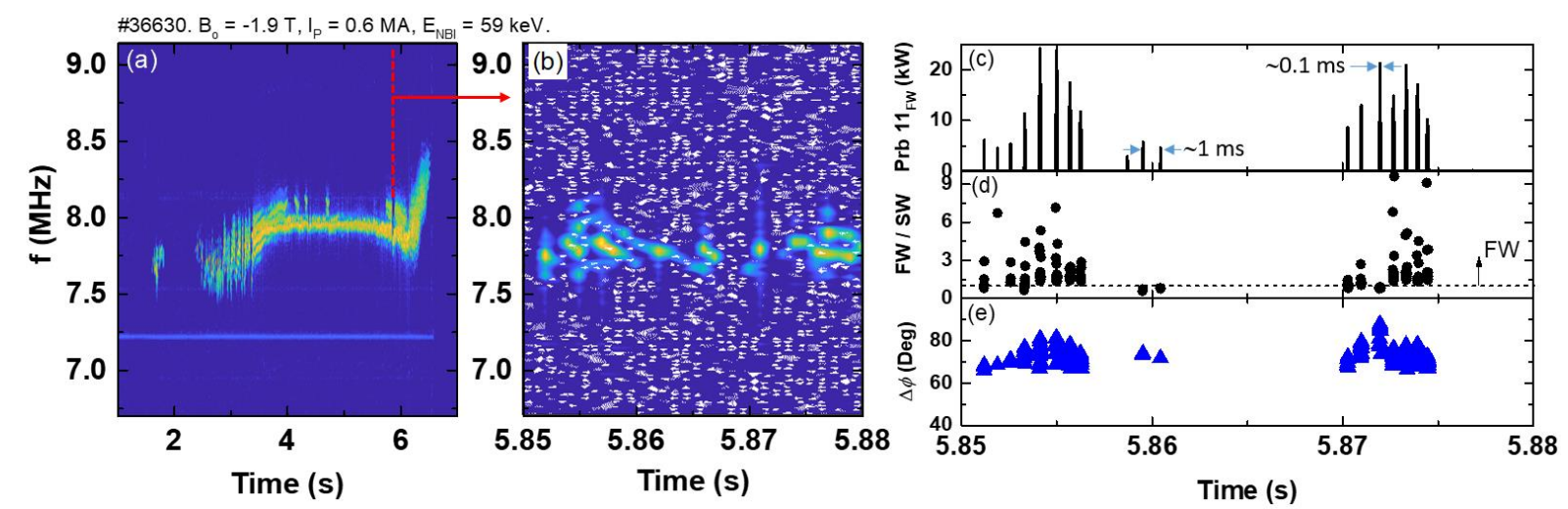

Fig. 9: Demonstration of high frequency Alfvén eigemodes in the presence of (sub-Alfvénic) beam ions only in discharge \#36630, $\mathrm{B}_{\mathrm{o}}=-1.9 \mathrm{~T}, \mathrm{I}_{\mathrm{P}}=0.6 \mathrm{MA}$. (a) a detailed spectral view of the mode; (b) a close-up view of the instability when the mode shows the least amount of spectral splitting; (c) the dominant (FW) amplitude of the mode; (d) the amplitude ratio of the FW to SW signal between two neighbouring probes; and (e) the phase difference between two neighbouring FW probes ( $\sim 0.06 \mathrm{~m}$ apart in the field-aligned direction). The NBI power stays constant at $2.5 \mathrm{MW}$ during emission. 


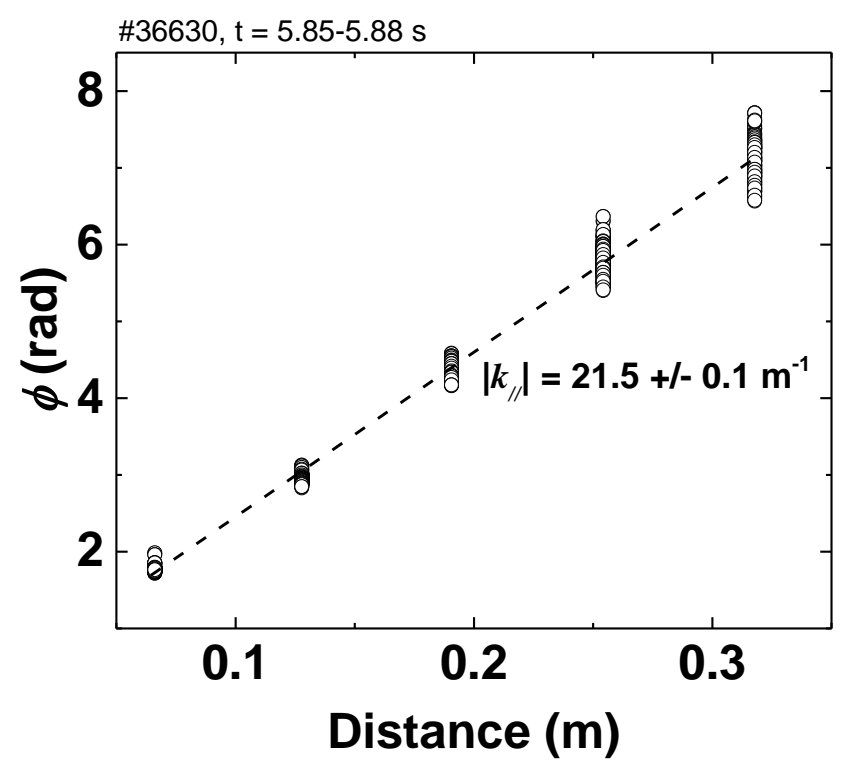

Fig. 10: The phase distribution $\phi$ along the HFB field-aligned probe array in discharge \#36630 at $\mathrm{t}=5.85-5.88 \mathrm{~s}$ [18]. The linear fit provides an estimate of the magnitude of the mode parallel wavenumber $\left|k_{/}\right|$. 

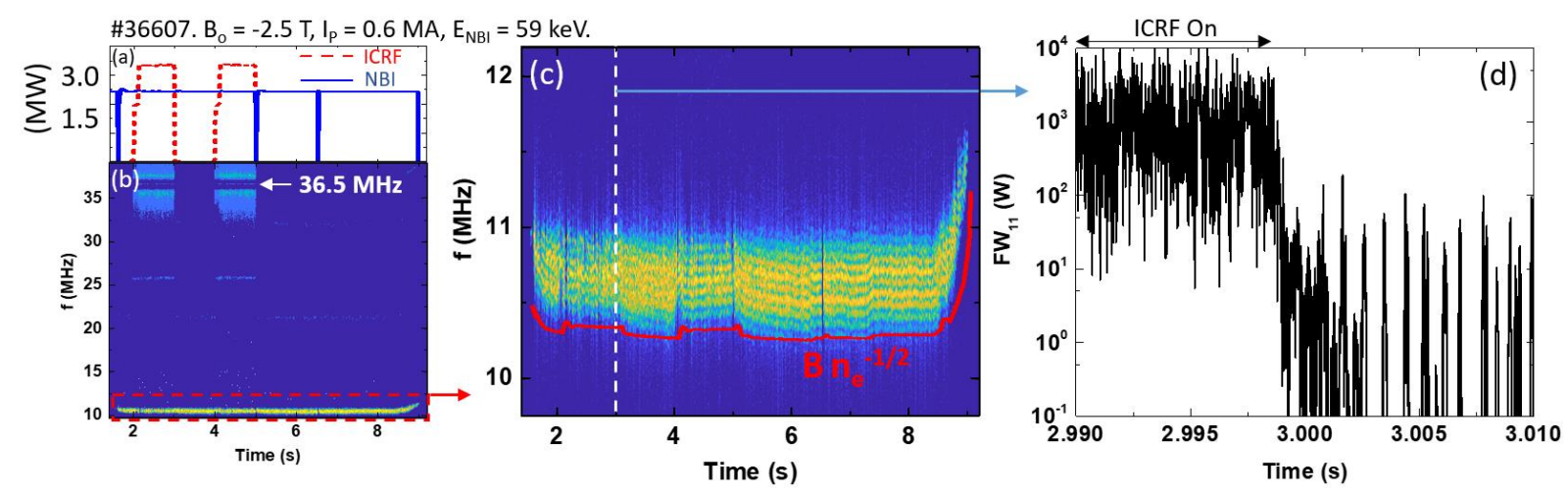

Fig. 11: A record long mode driven via sub-Alfvénic beam-injected ions. (a) The NBI and ICRF power; (b) a full frequency spectrum obtained by the ICE diagnostic; (c) a close-up view of the mode; and (d) the mode amplitude as detected by one of the FW probes (for t> $3.000 \mathrm{~s}$ ) and how it compares to the probe signal in the presence of $3.2 \mathrm{MW}$ of coupled ICRF power (for $\mathrm{t}<$ $2.998 \mathrm{~s})$. 

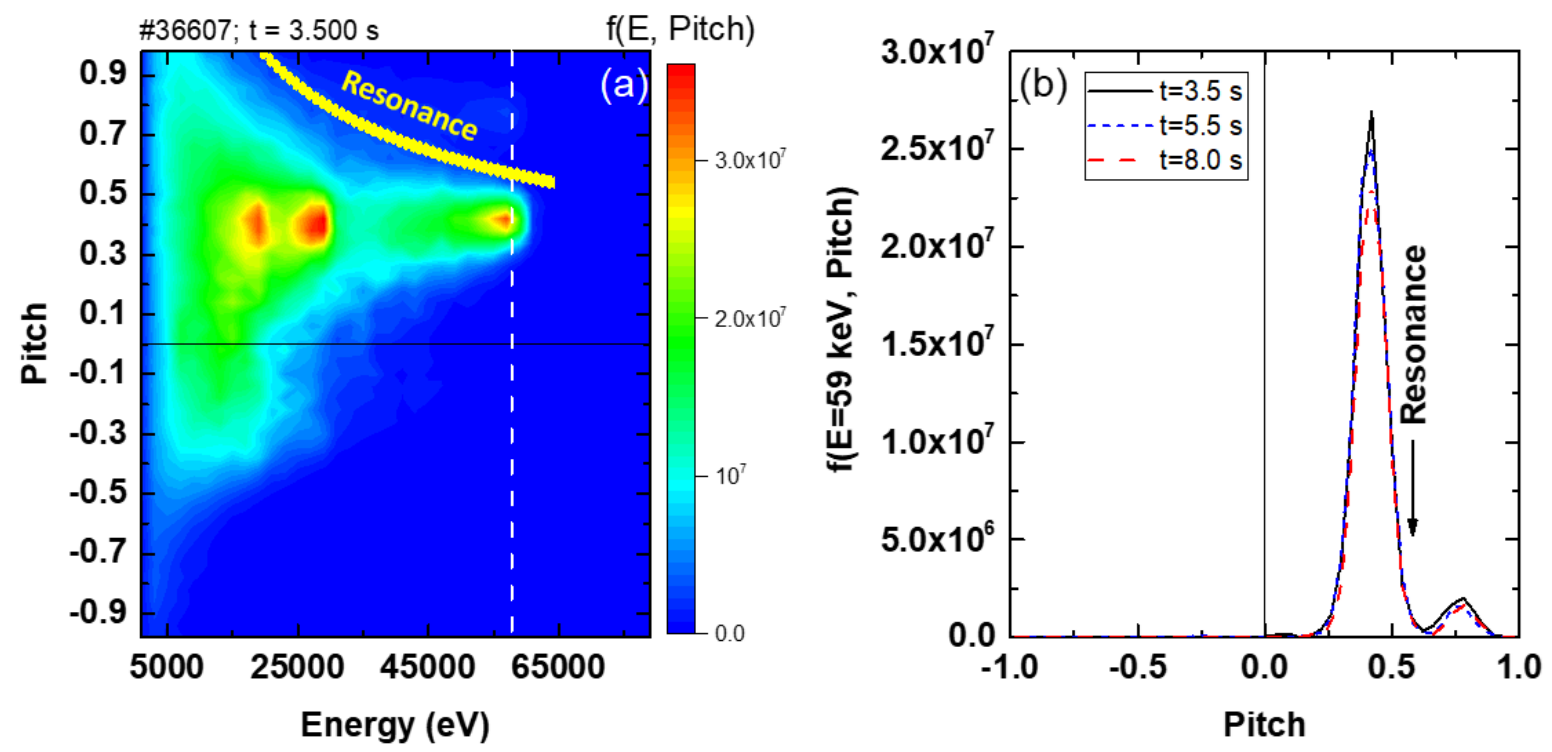

Fig. 12: (a) The beam ion distribution function $\mathrm{f}(\mathrm{E}, \mathrm{Pitch})$ at the radial location of the mode ( $\rho_{\text {tor }}$ $=0.7-0.8$ at the low-field side midplane), as determined by TRANSP [24]. The vertical white dashed line indicates the full energy component of the beam-injected ions (59 keV). (b) The beam ion distribution function $\mathrm{f}(\mathrm{E}, \mathrm{Pitch})$ at a constant beam injection energy $(\mathrm{E}=59 \mathrm{keV})$. The three lines in (b) correspond to three different times: $3.5 \mathrm{~s}$ (solid line), $5.5 \mathrm{~s}$ (short dashed line), and $8.0 \mathrm{~s}$ (dashed line) in discharge \#36607. The position of the local Doppler-shifted cyclotron resonance condition for our mode frequency $(10.8 \mathrm{MHz})$ and parallel wavenumber $\left(21.5 \mathrm{~m}^{-1}\right)$ is shown. 